\title{
Ion shock acceleration by large amplitude slow ion acoustic double layers in laser-produced plasmas
}

\author{
B. Eliasson \\ SUPA, Physics Department, John Anderson Building, \\ University of Strathclyde, Glasgow G4 ONG, UK \\ (Received 8 January 2013; Revised 5 February 2014)
}

\begin{abstract}
A kinetic model for the shock acceleration of ions in laser-produced plasmas is developed. A fraction of the warm ions are accelerated by the large amplitude monotonic potential of the shock created due the plasma compression and electron heating by the laser. The kinetic model for the monotonic shock is based on the slow ion acoustic double layer (SIADL). It is found that the amplitude of the large amplitude SIADL is almost uniquely defined by the electron temperature. Therefore, a balance between electron heating and plasma compression is needed for optimal ion acceleration by this scheme. Typical Mach numbers of the monotonic shocks are close to 1.5. The scheme could potentially produce monoenergetic ions with a relative energy spread of less than 1 percent. The model is compared with recent simulations and experiments, where efficient shocks acceleration and production of monoenergetic protons have been observed. Similarities and differences with other shock models are pointed out and discussed.
\end{abstract}

PACS numbers: 52.38.Kd,52.35.Tc,52.25.Dg

\section{INTRODUCTION}

Laser accelerated ions have important potential applications in the fields of cancer therapy [1-3], inertial confinement fusion [4], etc., where well defined energies of the protons are of great importance. A number of ion acceleration methods have been considered [5-7]. The interaction of an intense laser beam with an underdense plasma can lead to rapid heating of the electrons and acceleration of ions $[8,9]$. In target normal sheath acceleration (TNSA) [10-19], a minority species of light ions (typically protons) is accelerated by the hot sheath of laser-heated electrons normal to a solid target. The TNSA scheme has successfully been used to accelerate protons to several tens of $\mathrm{MeV}$, usually with a wide spectrum of energies [18]. In radiation pressure acceleration (RPA) [20-25], a thin target is accelerated by the radiation pressure of a circularly polarized laser, leading to a more peaked energy spectrum of the ions. The most severe problem that so far has prevented a greater success of the RPA scheme is the Rayleigh-Taylor instability $[26,27]$, which can rapidly disrupt the foil and broaden the ion energy spectrum [28]. Multi-ion species foils combined with different polarizations have been used to improve the energy spectrum of the accelerated protons [29, 30]. Shockrelated mechanisms for reflection and acceleration of ions in the contexts of space and laboratory plasmas have been investigated in the past [31-35], and go under names such as laminar electrostatic shocks, double layers, etc. Different types of double layers have been treated theoretically by Schamel and co-workers [36-38], including slow ion acoustic double layers (SIADLs) associated with the nonlinear slow ion acoustic mode, slow electron acoustic double layers (SEADLs)associated with the nonlinear slow electron acoustic mode, strong double layers [39] with beam-like free and trapped particle distributions, and current-free double layers [40]. A different scheme that has gained interest is combined radiation pressure and shock or soliton acceleration of ions [41-46]. In this scheme, the laser is injected towards an over-dense gas target, which leads to a compression of the plasma and heating of the electrons. This can result in a large amplitude shock-like or soliton-like structure [46] that propagates into the target. Due to the large amplitude electrostatic potential, a portion of the upstream ions are specularly reflected by the nonlinear structure. Since the target is over-dense, the shock or soliton does not interact directly with the laser light after the initial compression of the plasma.

Recent kinetic shock models either treat the ions as a cold fluid $[47,48]$ or kinetically with a finite momentum spread $[49,50]$, while the electrons are divided into free and trapped/reflected downstream populations. Shocks with oscillatory downstream shock potentials have been found by including hot ions and considering trapped downstream electron populations and a population of reflected upstream ions $[45,49,50]$. The aim is here to develop a kinetic model of monotonic (non-oscillatory) shock structures in un-magnetized plasmas, including hot electrons and ions, and to investigate the conditions for the existence of monotonic shocks on the plasma parameters. 


\section{KINETIC SHOCK MODEL}

Our model covers the structure of the shock, and how the ions are reflected by the self-consistent large amplitude potential. A solution of the time-independent ion Vlasov equation

$$
v \frac{\partial f_{i}}{\partial x}-\frac{\partial \phi}{\partial x} \frac{\partial f_{i}}{\partial v}=0
$$

is given by $f_{i}(\mathcal{E})$ where

$$
\mathcal{E}=\frac{v^{2}}{2}+\phi-\phi_{\max }
$$

is the conserved energy of an ion moving with velocity $v$ under the influence of the electrostatic potential $\phi$. We assume that the potential is at $\phi=\phi_{\max }$ at $x=-\infty$, and drops at the double layer so that $\phi=0$ at $x=+\infty$, where the ion distribution contains reflected ions. In Eqs. (1) and (2), the velocity $v$ has been normalized by the ion thermal velocity $v_{T i}=\sqrt{k_{B} T_{i} / m_{i}}$, the potentials $\phi$ and $\phi_{\max }$ by $k_{B} T_{i} / e$, space $x$ by $v_{T i} / \omega_{p i}$ where $\omega_{p i}=\sqrt{n_{0} e^{2} /\left(\epsilon_{0} m_{i}\right)}$ is the ion plasma frequency, and the ion distribution function $f_{i}$ by $n_{0} / v_{T i}$. Here $T_{i}$ and $n_{0}$ is the ion temperature and number density, respectively, at $x=-\infty$. Furthermore, $k_{B}$ is Boltzmann's constant, $\epsilon_{0}$ is the electric vacuum permittivity, $e$ is the magnitude of the elementary charge, and $m_{i}$ is the ion mass. Ions with $\mathcal{E}>0$ are "free" and can penetrate the shock, while ions with $\mathcal{E}<0$ are reflected by the shock potential. Our model is based on the one-dimensional Vlasov-Poisson system using Schamel's theory of SIADLs [38] to construct well-behaved distribution functions. In the frame of the shock, moving with a velocity $u_{i 0}$ along the $x$-axis, the equilibrium ions at $x=-\infty$ obey a shifted Maxwellian distribution function $f_{i}=(1 / \sqrt{2 \pi}) \exp \left[-\left(v+u_{i 0}\right)^{2} / 2\right]$ streaming in the opposite direction. The particular choice of downstream ion distribution seems to be quite consistent with the recent simulation results of laser produced shocks in Ref. [43] (see their Fig. 4c-d). This is fulfilled by the special choice of ion velocity distribution function $[38,51]$

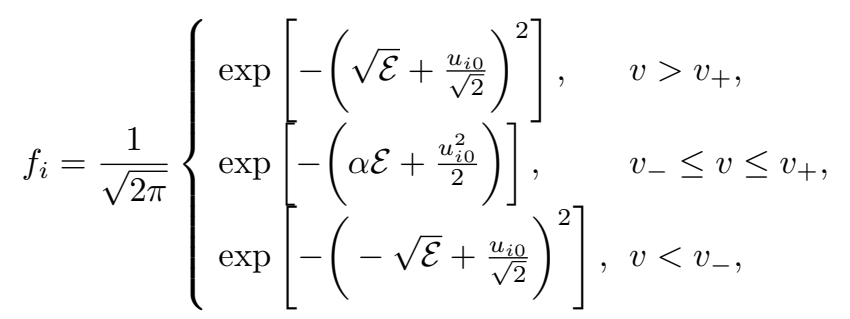

where $v_{ \pm}= \pm 2^{1 / 2}\left(\phi_{\max }-\phi\right)^{1 / 2}$ defines the limit $\mathcal{E}=0$ between the reflected $(\mathcal{E}<0)$ and free $(\mathcal{E}>0)$ ions in velocity space, and $u_{i 0}$ is the velocity of the double layer compared to the mean velocity of the ions at $x=-\infty$. The free ions are divided into two populations, one which overtakes the shock $\left(v>v_{+}\right)$, and one which is overtaken by the shock $\left(v<v_{-}\right)$, hence $f_{i}$ is double-valued for $\mathcal{E}>0$. The reflected ions in the range $v_{-} \geq v \geq v_{+}$are characterized by the parameter $\alpha$, where $\alpha=0$ gives a flat-topped reflected ion population, $\alpha<0$ gives a beam-like, excavated population in velocity space, and $\alpha=-\infty$ gives a completely depleted distribution with no reflected ions. The parameter $\alpha$ is sometimes referred to as the "trapping parameter" in the context of ion holes [38] to describe trapped particle distributions. The ion density $n_{i}$ (normalized by $n_{0}$ ) is obtained by integrating Eq. (3) over velocity space and can be expressed in terms of special functions as $[36,52]$

$$
n_{i}\left(\phi, \phi_{\max }\right)=\exp \left(-\frac{u_{i 0}^{2}}{2}\right)\left\{I\left(\phi_{\max }-\phi\right)+K\left(\frac{u_{i 0}^{2}}{2}, \phi_{\max }-\phi\right)+\frac{2}{\sqrt{\pi|\alpha|}} W\left[\sqrt{\alpha\left(\phi-\phi_{\max }\right)}\right]\right\}
$$

where $I(\Phi)=\exp (\Phi)[1-\operatorname{erf}(\sqrt{\Phi})], \operatorname{erf}(u)$ is the error function,

$$
K(X, \Phi)=\frac{2}{\sqrt{\pi}} \int_{0}^{\pi / 2} \sqrt{X} \cos (\Phi) \exp \left(-\Phi \tan ^{2} \theta+X \cos ^{2} \theta\right) \operatorname{erf}(\sqrt{X} \cos \theta) d \theta
$$

and

$$
W(u)=\exp \left(-u^{2}\right) \int_{0}^{u} \exp \left(t^{2}\right) d t
$$


is Dawson's integral. The electrons are assumed to be isothermal so that the electron number density obeys the Boltzmann distribution of the form [45]

$$
n_{e}\left(\phi, \phi_{\max }\right)=n_{i}\left(0, \phi_{\max }\right) \exp (\phi / \theta)
$$

where $\theta=T_{e} / T_{i}$. Equation (7) ensures that $n_{e}=n_{i}$ when $\phi=0$. It should be noted that kinetic models for the electrons have been used both in the non-relativistic [32, 47] and relativistic [49, 50] regimes; to obtain Eq. (7), we have made the simplifying assumption that the electrons are thermalized on the ion time-scale and assume a nonrelativistic Maxwell-Boltzmann distribution. Inserting the expressions for the ion and electron densities into Poisson's equation, we have

$$
\frac{d^{2} \phi}{d x^{2}}=n_{e}\left(\phi, \phi_{\max }\right)-n_{i}\left(\phi, \phi_{\max }\right)
$$

By defining the Sagdeev potential

$$
V\left(\phi, \phi_{\max }\right)=\int_{0}^{\phi}\left[n_{i}\left(\phi^{\prime}, \phi_{\max }\right)-n_{e}\left(\phi^{\prime}, \phi_{\max }\right)\right] d \phi^{\prime}
$$

we have from Poisson's equation

$$
\frac{d^{2} \phi}{d x^{2}}=-\frac{\partial V}{\partial \phi}
$$

which, multiplying by $d \phi / d x$, gives

$$
\frac{1}{2}\left(\frac{d \phi}{d x}\right)^{2}=-V
$$

For the existence of a laminar shock, the conditions are that both $V=0$ and $\partial V / \partial \phi=0$ for $\phi=0$ and $\phi=\phi_{\max }$, and that $V<0$ for $0<\phi<\phi_{\max }$. In particular, the condition

$$
V\left(\phi_{\max }, \phi_{\max }\right)=0
$$

defines the amplitude of the shock, while it holds trivially from Eq. (9) that $V\left(0, \phi_{\max }\right)=0$. The condition $\partial V / \partial \phi=0$ at $\phi=\phi_{\max }$ is equivalent to the quasi-neutrality condition $n_{e}=n_{i}$ at $x=-\infty$, where it also holds that $n_{i}\left(\phi_{\max }, \phi_{\max }\right)=1$. Using this together with the expression (7) for the electron density, we have

$$
n_{i}\left(0, \phi_{\max }\right)=\exp \left(-\phi_{\max } / \theta\right) .
$$

The conditions (12) and (13) give $\phi_{\max }$ and $u_{i 0}$ for given values of $\theta$ and $\alpha$. After these have been found, Eq. (11) can be integrated to obtain the spatial profile of the shock potential. It should be noted that the charge neutrality condition (13) leads to monotonic shocks, in contrast to the models of oscillatory shocks $[45,49,50]$ where different boundary conditions were used.

\section{NUMERICAL RESULTS}

Figures $1 \mathrm{a}-\mathrm{d}$ show solutions of Eqs. (12) and (13) in the $\left(u_{i 0}, \phi_{\max }\right)$-plane for $\theta=10$ and different values of $\alpha$. Simultaneous solutions of Eqs. (12) and (13) are found where the two curves intersect each other. These solutions are associated with the nonlinear slow ion acoustic mode (discussed below), and the corresponding double layers are referred to as slow ion acoustic double layers (SIADLs) in the classification scheme of Schamel [36-38]. We see in Figs. 1a-d that the system has solutions for both large and small values of $\phi_{\max }$, which could indicate the existence of double layers. However, the small amplitude solutions seen in Figs. $1 \mathrm{a}-\mathrm{d}$ (for $\phi_{\max }<2$ ) produce $V>0$ in the interval $0<\phi<\phi_{\max }$ and hence do not permit double layers. The existence of small amplitude, weak double layers requires non-Maxwellian electrons which are more flat-topped or excavated $[37,53]$. The value $\alpha=-0.9393$ is close to the maximum negative limit, beyond which no large amplitude solutions exist. The double layers can be seen as limiting cases of ion hole solutions, (see Fig. 6 of Ref. [52]), where the maximum negative $\alpha$ corresponds to a current-free double layer. The condition that $V<0$ in the interval $0<\phi<\phi_{\max }$ further restricts the existence of double layers. It turns out that $\alpha$ must have larger negative values than about -0.633 for laminar shocks to exist, as illustrated in 
a)

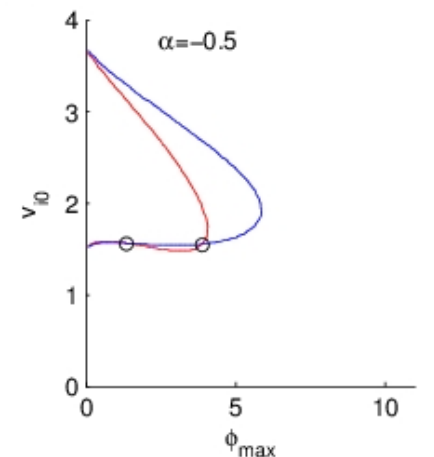

e)

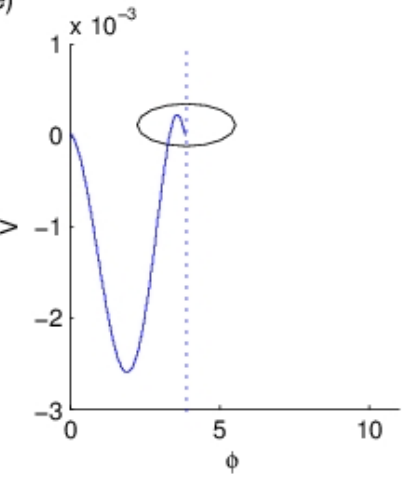

b)

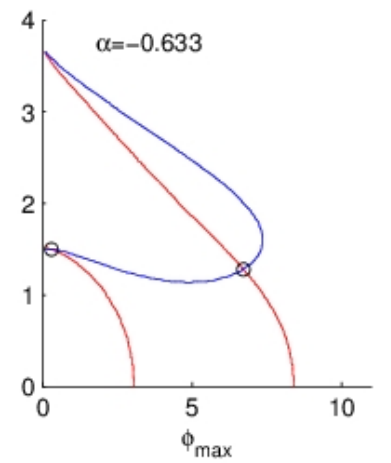

f)

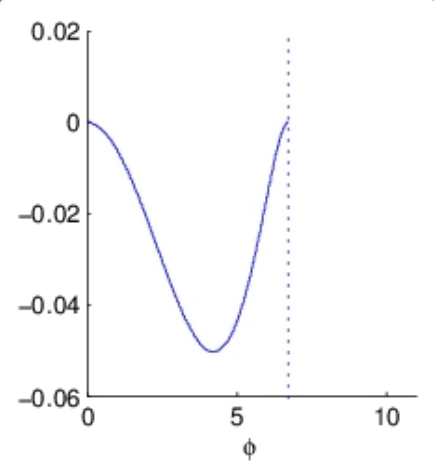

c)

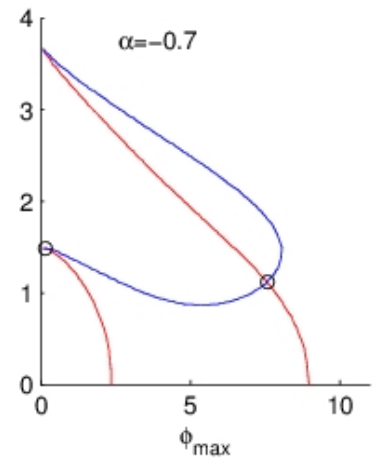

g)

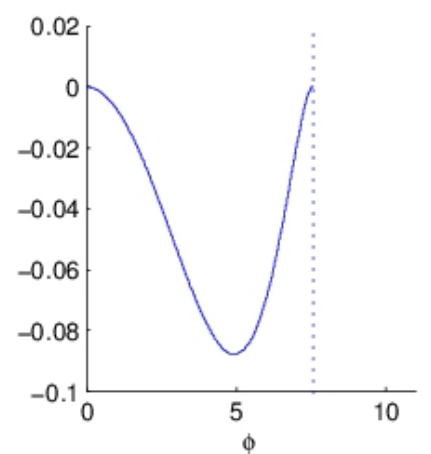

d)

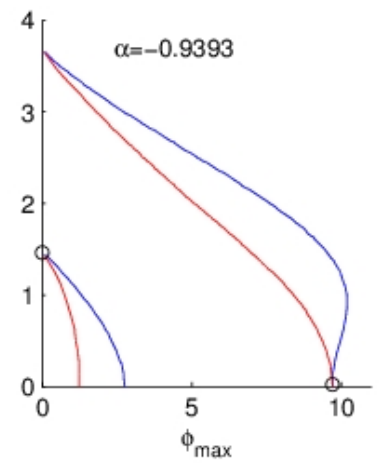

h)

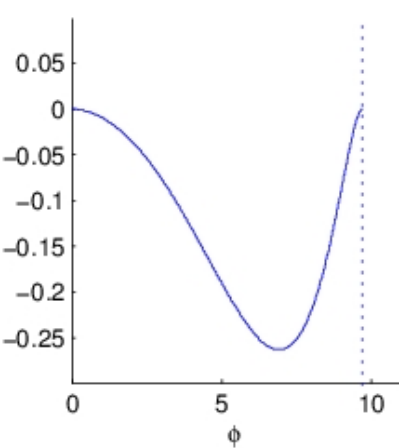

FIG. 1: (color online) a)-d): Solutions of Eqs. (12) and (13) (blue and red curves, respectively) in the $\left(\phi_{\max }, u_{i 0}\right)$-plane for $\theta=10$ and different values of $\alpha$. Where the curve intersect each other (indicated with circles), both equations are simultaneously solved, indicating possible double layer solutions. The large $\phi_{\max }$ solutions are a) $\left(u_{i 0}, \phi_{\max }\right)=(1.5467,3.8935)$ for $\alpha=-0.5, \mathrm{~b})\left(u_{i 0}, \phi_{\max }\right)=(1.2785,6.7222)$ for $\left.\alpha=-0.633, \mathrm{c}\right)\left(u_{i 0}, \phi_{\max }\right)=(1.1149,7.5699)$ for $\alpha=-0.7$, and $\left.\mathrm{d}\right)$ $\left(u_{i 0}, \phi_{\max }\right)=(0.0147,9.70235)$ for $\alpha=-0.9393$. The corresponding Sagdeev potentials are shown in panels e)-f). The vertical dotted lines indicate $\phi=\phi_{\max }$. For $\alpha>-0.633$ the shock solution is not permitted, since in this case $V>0$ part of the interval $0<\phi<\phi_{\max }$ [e.g. in panel e) $\alpha=-0.5$ indicated by an ellipse], while $-0.633>\alpha>-0.9393$ permit shock solutions [panels f) - h)]

Figs. 1e-h. For smaller values, such as $\alpha=-0.5$ (cf. Fig. 1e), $V$ is larger than zero in part of the interval and prevents the existence of double layers. Equations (12) and (13) also have simultaneous solutions in the linear limit $\phi_{\max } \rightarrow 0$. In this limit, the phase velocity $u_{i 0}=v_{p h}$ is given by the dispersion relation for undamped ion acoustic waves in the long-wavelength limit (omitting Landau damping by keeping only the principal part of the Landau contour integral),

$$
1-\pi v_{p h} H\left[f_{i 0}\left(v_{p h}\right)\right]+1 / \theta=0
$$

where

$$
H\left[f_{i 0}\left(v_{p h}\right)\right]=-\frac{1}{\pi} \int_{0}^{\infty} \frac{f_{i 0}\left(v_{p h}+u\right)-f_{i 0}\left(v_{p h}-u\right)}{u} d u
$$

is the Hilbert transform of the Maxwellian equilibrium ion distribution function $f_{i 0}(v)=(2 \pi)^{-1 / 2} \exp \left(-v^{2} / 2\right)[54]$. The dispersion relation has one high-velocity solution associated with the usual linear ion acoustic mode $v_{p h} \approx \sqrt{3+\theta}$, and one low-velocity solution associated with the slow ion acoustic mode $[38,55] v_{p h} \approx 1.305\left(1+\theta^{-1}\right)$, for $\theta \gg 1$. For $\theta=10$ we find numerically from the dispersion relation (14) and from Figs. 1a-d that the velocity of the slow mode is given by $v_{p h}=1.45$ and the fast ion acoustic mode $v_{p h}=3.72$. The fast and slow ion acoustic modes merge for $\theta \approx 3.5$, and for smaller values of $\theta$ the undamped ion acoustic modes cease to exist (see e.g. Ref. [55]). It should be noted that the existence criterion for finite amplitude ion holes and SIADLs are [52] $u_{i 0}<1.305\left(1+\theta^{-1}\right), \theta>3.5$, and $\alpha<0$ (strictly negative).

The spatial profiles of the electrostatic shock potential are plotted in Fig. 2a for the cases $\alpha=-0.633,-0.7$ and -0.9393. The laminar shocks exhibit a jump from its high voltage value close to $\phi_{\max }$ at the left boundary to near zero at the right boundary. The corresponding ion distribution functions, shown in Figs. 2b-d, are close to Maxwellian at 
a)

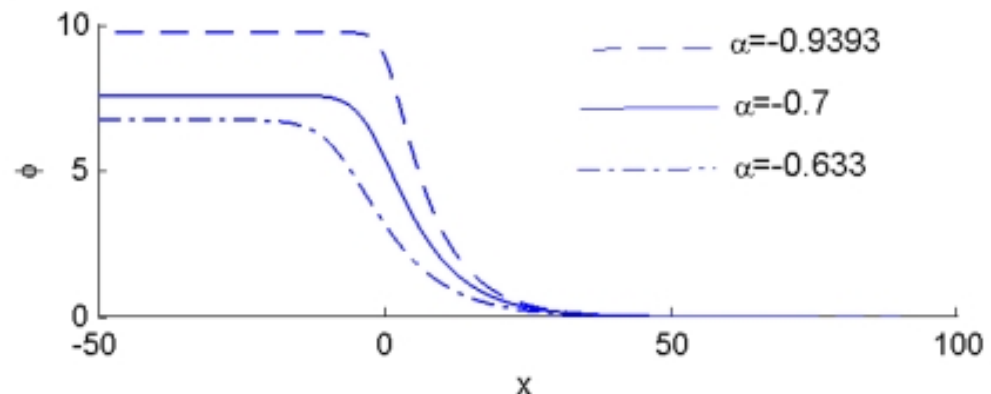

b)

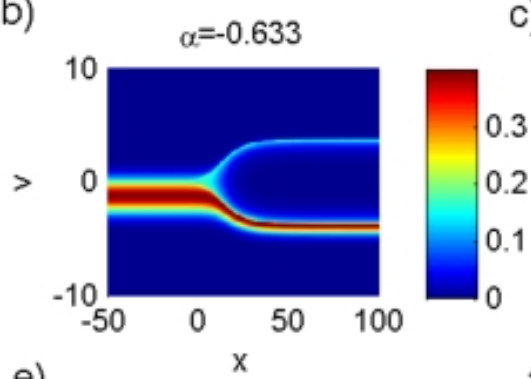

e)

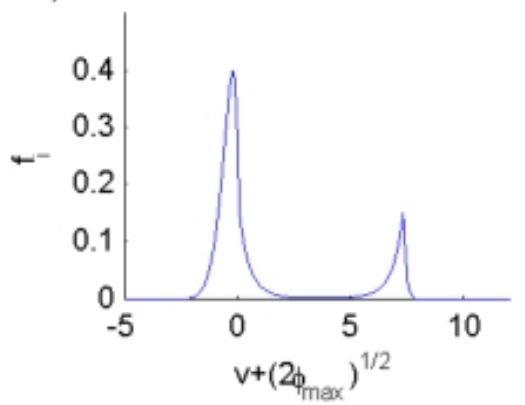

c)

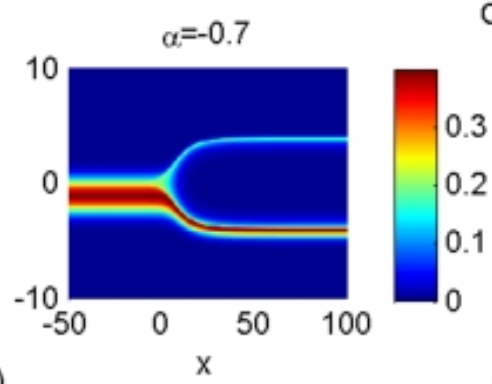

f)

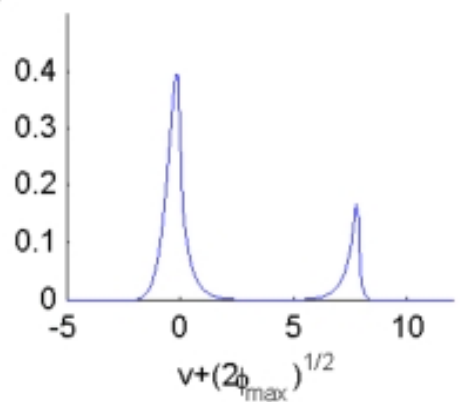

d)

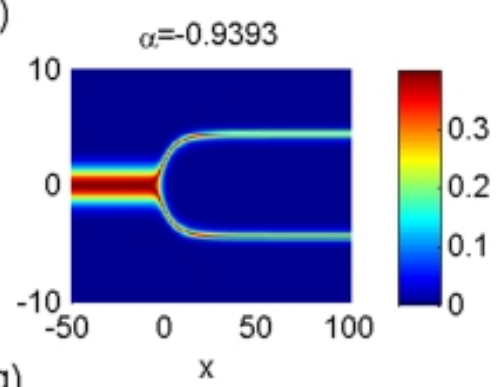

g)

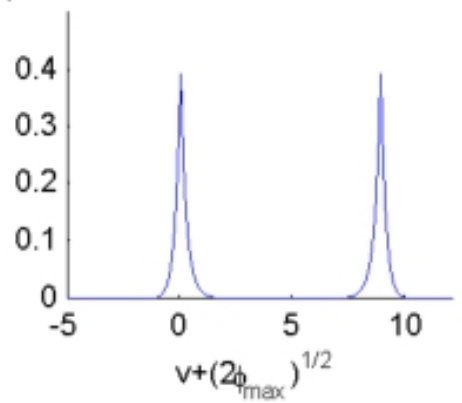

FIG. 2: a) Profiles of the electrostatic potential for $\theta=10$ and $\alpha=-0.633$ (dash-dotted curve), $\alpha=-0.7$ (solid curve), and $\alpha=-0.9393$ (dashed curve). b) -d): The ion distribution function $f_{i}(x, v)$ for b) $\left.\alpha=-0.633, \mathrm{c}\right) \alpha=-0.7$, and d) $\alpha=-0.9393$. e) $-\mathrm{g})$ : The corresponding upstream ion distribution function in the frame of the incoming plasma. A beam is seen with a relative speed of approximately $2\left(2 \phi_{\max }\right)^{1 / 2}$, corresponding to an ion energy of about $4 \phi_{\max }$ relative to the upstream ions, which can be assume to be at rest in the laboratory frame. The relative energy spread of the beam is $1.0 \times 10^{-2}, 6.3 \times 10^{-3}$ and $1.7 \times 10^{-3}$ for the respective cases e)-g), with a relative beam density of $0.24,0.29$ and 1.0, respectively, compared to the upstream ion density.

the left boundary, while it splits into two populations of ions at the right boundary with upstream, incoming ions with negative velocities and a significant portion of reflected ions with positive velocities. The limiting large amplitude value $\alpha=-0.9393$ gives rise to two symmetric ion populations with equal incoming and reflected densities. In Figs. $2 \mathrm{e}-\mathrm{g}$, we show the ion velocity distribution at the right-hand boundary, shifted to a frame moving with the upstream incoming ions (with negative velocities in Figs. $2 \mathrm{~b}-\mathrm{d}$ ). The reflected beam has a velocity close to $2\left(2 \phi_{\max }\right)^{1 / 2}$, corresponding to an ion kinetic energy of $4 \phi_{\max }$. It should be emphasized that only large amplitude shock solutions exist, for a finite range of $\alpha$ in the interval $-0.633>\alpha>-0.9393$ and with a corresponding amplitude of the shock potential in the range $6.72<\phi_{\max }<9.7$. The shock speed (the downstream bulk speed minus the upstream, incoming beam speed) can be estimated as $v_{\text {shock }}=\sqrt{2 \phi_{\max }}-u_{i 0}$ and lies in the range $2.38<v_{\text {shock }}<4.4$. Dividing with the linear ion acoustic speed $v_{p h} \approx \sqrt{\theta} \approx 3.2$ gives a Mach number in the range $0.75<M<1.4$. The relative energy spread of the beam ions is about $1 \%$ or less.

Recent experiments and simulation studies [43, 44] have shown efficient shock acceleration of protons using thick gas targets. The plasma is initially compressed by radiation pressure acceleration, the electrons are heated, and a shock is formed propagating into the overdense plasma. A beam of reflected ions with a narrow energy spectrum is subsequently formed. In the simulation study of Haberberger et al. [43], the estimated electron temperature was 
a)

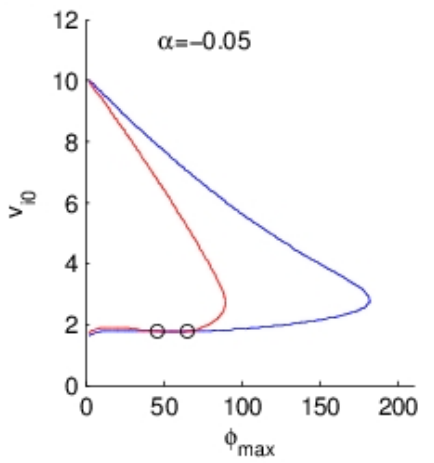

e)

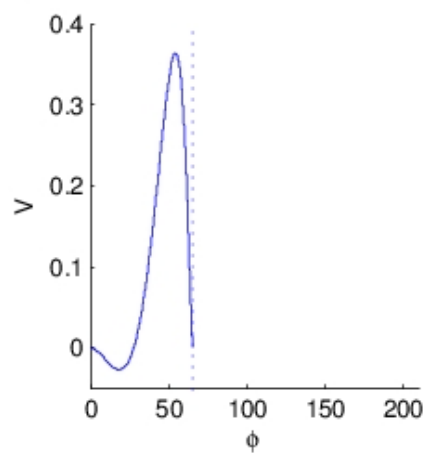

f) b)
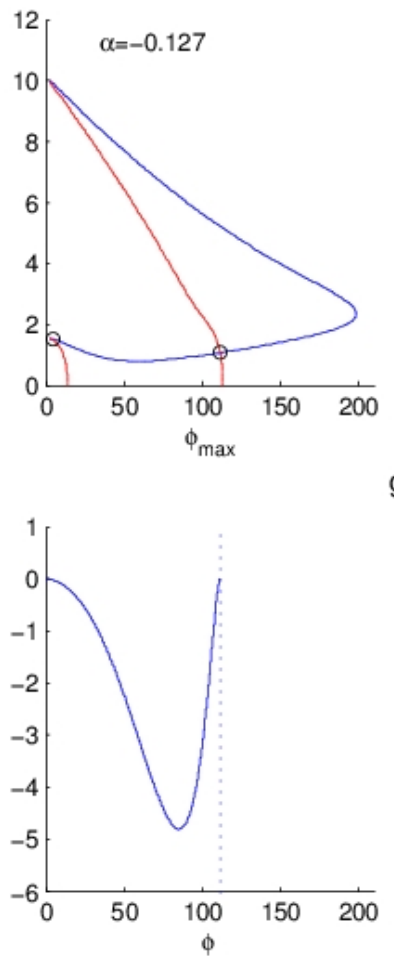

c)

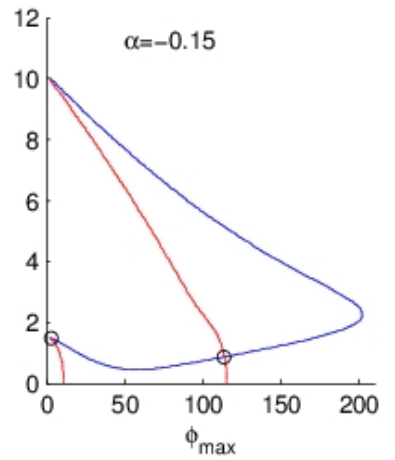

g)

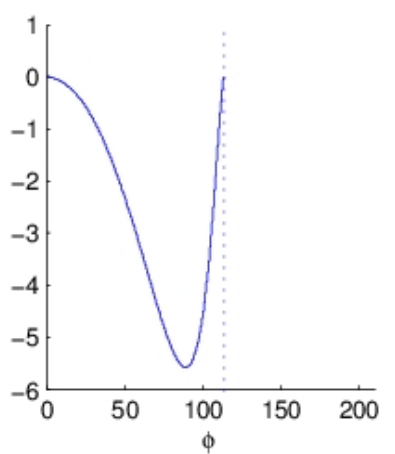

d)

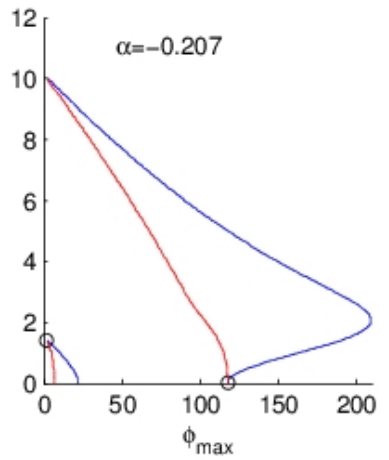

h)

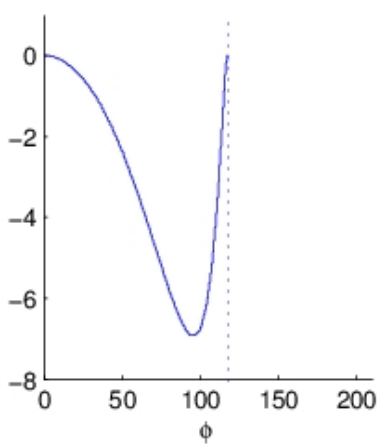

FIG. 3: a)-d): Solutions of Eqs. (12) and (13) (blue and red curves, respectively) in the $\left(\phi_{\max }, u_{i 0}\right)$-plane for $\theta=100$ and different values of $\alpha$. Where the curve intersect each other (indicated with circles), both equations are simultaneously solved, indicating possible double layer solutions. The large $\phi_{\max }$ solutions are a) $\left(u_{i 0}, \phi_{\max }\right)=(1.7651,65.3327)$ for $\alpha=-0.05$, b) $\left(u_{i 0}, \phi_{\max }\right)=(1.0686,111.5314)$ for $\left.\alpha=-0.127, \mathrm{c}\right)\left(u_{i 0}, \phi_{\max }\right)=(0.87084,113.8435)$ for $\alpha=-0.15$, and $\left.\mathrm{d}\right)\left(u_{i 0}, \phi_{\max }\right)=$ $(0.022987,117.5444)$ for $\alpha=-0.207$. The corresponding Sagdeev potentials are shown in panels e)-f). The vertical dotted lines indicate $\phi=\phi_{\max }$. For $\alpha>-0.127$ the shock solution is not permitted, since in this case $V>0$ part of the interval $0<\phi<\phi_{\max }$ [e.g. in panel e) $\alpha=-0.05$ ], while $-0.127>\alpha>-0.207$ [panels f)-h)] allow shock solutions.

about 100 times larger than the upstream ion temperature, corresponding to $\theta=100$. In their Fig. 4, they observed a significant fraction of reflected ions. The upstream, expanding ions had a velocity of $0.1 c$ relative to the laboratory frame, and the shock-reflected beam had a velocity of $0.2 c$, corresponding to an ion kinetic energy of about $20 \mathrm{MeV}$ relative to the laboratory frame. As a comparison with these studies, show in Figs. 3a-d solutions of Eqs. (12) and (13) in the $\left(u_{i 0}, \phi_{\max }\right)$-plane for $\theta=100$ and different values of $\alpha$. We see in Figs. 3a-d that the system again has solutions for both large and small values of $\phi_{\max }$, of which it turns out that the small amplitude solutions do not support double layers since $V$ takes positive values in the interval $0<\phi<\phi_{\max }$. The value $\alpha=-0.207$ is close to the maximum amplitude limit, above which no shock solutions exist, and $\alpha$ must have larger negative values than about -0.127 for laminar shocks to exist; see Figs. $3 \mathrm{f}-\mathrm{h}$. For smaller values, such as $\alpha=-0.05$ (cf. Fig. 4 e), $V$ is larger than zero in part of the interval and prevents the existence of double layers.

The associated profiles of the electrostatic potential are plotted in Fig. 4a, and the ion distribution function is plotted in Figs. 4b-d where it is seen that a significant portion ions are reflected by the shock. Here, the limiting large amplitude value $\alpha=-0.207$ gives rise to two symmetric populations of incoming and reflected ions with equal densities. In the frame moving with the upstream ions, shown in Figs. $4 \mathrm{e}-\mathrm{g}$, the reflected beam has a velocity close to $2\left(2 \phi_{\max }\right)^{1 / 2}$, corresponding to an ion kinetic energy of $4 \phi_{\max }$. Only large amplitude solutions exist, for a finite range of $\alpha$ in the interval $-0.127>\alpha>-0.207$ with a corresponding amplitude of the shock potential in the range $111.5<\phi_{\max }<117.5$. Using an electron temperature of $1 \mathrm{MeV}$ and an ion temperature of $10 \mathrm{keV}$, the ion beam energy $\approx 4 \phi_{\max } \approx 450$ in dimensionless units corresponds in dimensional units to about $4.5 \mathrm{MeV}$ and a relative beam speed of $0.07 c$, calculated in the frame of the upstream ions. In a laboratory frame where the upstream, expanding ions have velocity of $0.1 c$, the beam speed is $0.17 c$ and the energy of the reflected ions are about $13 \mathrm{MeV}$. This is comparable to, although somewhat lower than, the simulation results in Ref. [43] where ion energies up to $20 \mathrm{MeV}$ were observed. The higher ion energies in the simulations could be explained by an additional sheath acceleration of the ions due to a finite size of the plasma, which is not taken into account in the present theoretical model. We 
a)

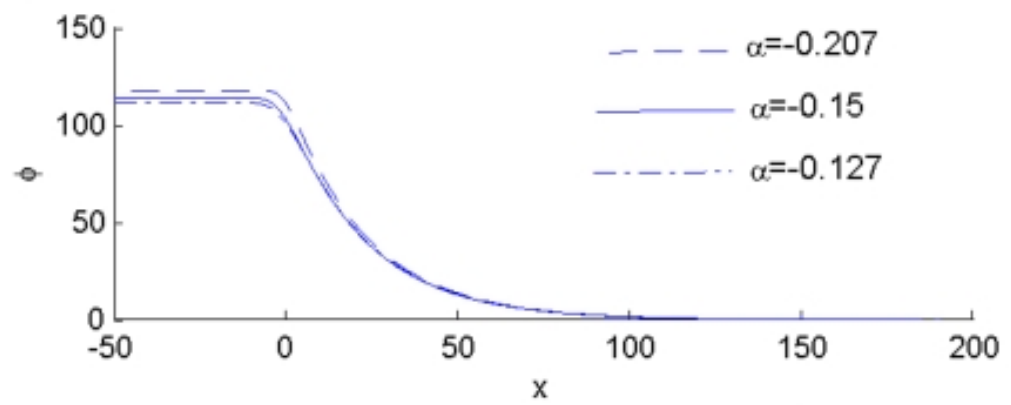

b)

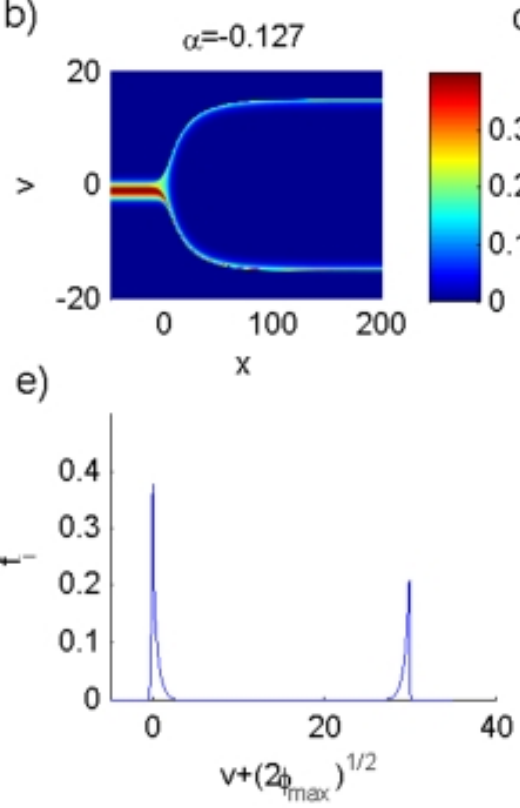

c)

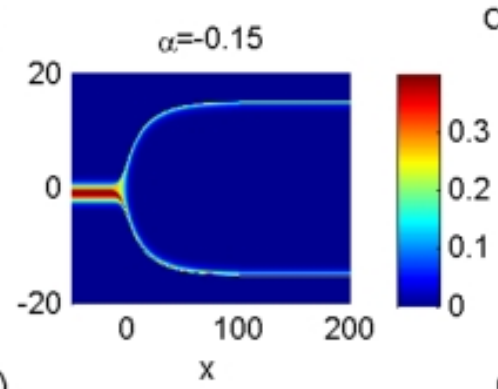

f)

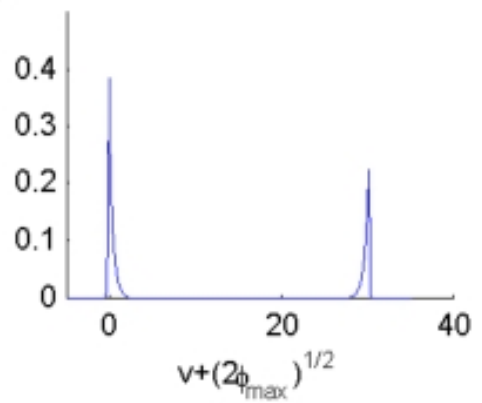

d)

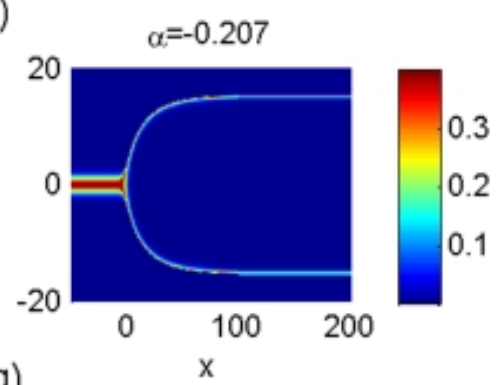

g)

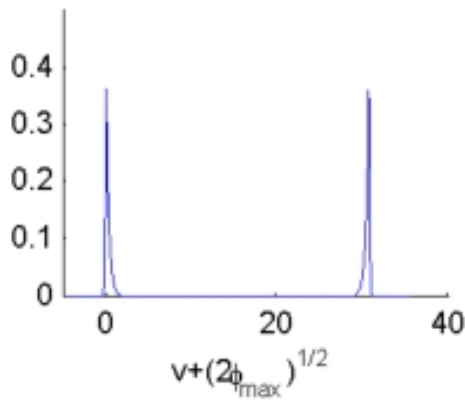

FIG. 4: a) Profiles of the electrostatic potential for $\theta=100$ and $\alpha=-0.127$ (dash-dotted curve), $\alpha=-0.15$ (solid curve), and $\alpha=-0.207$ (dashed curve). b) -d): The ion distribution function $f_{i}(x, v)$ for b) $\left.\alpha=-0.127, \mathrm{c}\right) \alpha=-0.15$, and d) $\alpha=-0.207$. e)-g): The corresponding upstream ion distribution function in the frame of the incoming plasma. A beam is seen with a relative speed of approximately $2\left(2 \phi_{\max }\right)^{1 / 2}$, corresponding to an ion energy of about $4 \phi_{\max }$ relative to the upstream ions, which can be assume to be at rest in the laboratory frame. The relative energy spread of the beam is $4.1 \times 10^{-4}, 2.6 \times 10^{-4}$ and $1.24 \times 10^{-4}$ for the respective cases e)-g), with a relative beam density of $0.65,0.67$ and 1.0 , respectively, compared to the upstream ion density.

observe that the shock speed $v_{\text {shock }} \approx \sqrt{2 \phi_{\max }}-u_{i 0}$ is in the narrow range $14.0<v_{\text {shock }}<15.3$, which gives a Mach number $1.4<M<1.5$ using the linear ion acoustic speed $v_{p h} \approx \sqrt{\theta}=10$. Here the relative energy spread of the beam ions is less than $0.1 \%$.

An interesting observation is that the monotonic shocks have both a maximum and minimum amplitude for a given value of the electron-to-ion temperature ratio $\theta$. For large values of $\theta$, the shock potential can only take a narrow range of large-amplitude values, as seen in Figs. 3 and 4 . To a good approximation, we have $\phi_{\max } / \theta \approx 1$ for $\theta$ ranging from 10 to 100 . Using the shock velocity $v_{\text {shock }} \approx \sqrt{2 \phi_{\max }} \approx \sqrt{2 \theta}$ and dividing with the linear ion acoustic speed $v_{p h} \approx \sqrt{\theta}$ gives the Mach number $M \approx \sqrt{2} \approx 1$.4. In dimensional units, the shock potential $\phi_{\max }$ is approximately equal to $k_{B} T_{e} / e$ for the existence of the monotonic shock. The ratio of the upstream to the downstream electron density is close to $\exp \left(e \phi_{\max } / k_{B} T_{e}\right) \approx 2.7$, and, since the upstream ions consist of and almost equal amount of reflected and incoming ions, the ratio between the downstream (incoming) ion density to the upstream ion density is approximately 5.4. This determines the optimal rate of compression of the plasma for the existence of the laminar shock. Hence, for laser compression, there should be a balance $P_{r a d}=P_{e}$, where $P_{r a d}=2 I_{0} / c$ is the radiation pressure $\left(I_{0}\right.$ is the laser intensity and $c$ the speed of light) and $P_{e}=n_{0} k_{B} T_{e}$ is the electron pressure, taking into account that $n_{0}$ is about $5-6$ times larger than the downstream density. If the laser amplitude is too small, there will not be a well-defined double 
layer, since there is a predicted minimum amplitude of the double layer. If the laser amplitude is too large, the laser will work as a piston and will always be in contact with the shock front, similar to the cold ion piston case discussed in Ref. [32], and no double layer will develop.

\section{SUMMARY}

In summary, we have presented a kinetic model for the shock acceleration of ions in laser-produced plasmas, where the large amplitude laser is heating the electrons and compressing the overdense plasma via the radiation pressure. The large amplitude shock specularly reflects a large amount of the downs-stream ions. It is found from the kinetic model that the amplitude of the large amplitude shock is almost uniquely defined by the electron temperature via $\phi_{\max } \approx k_{B} T_{e} / e$, leading to an approximate ratio of 5-6 between the upstream and downstream ion densities. Hence the model predicts an optimal balance between plasma compression and electron heating for mono-energetic ion beams to be produced. The balance between the laser radiation pressure and the downstream electron thermal pressure then gives the optimal laser intensity for producing monoenergetic (potentially less than $1 \%$ energy spread) ions via monotonic double layers/shocks. Typical Mach numbers of the monotonic shock are close to $M \approx 1.5$. The analytic model agrees reasonably well with recent simulations and experiments [43], where efficient shocks acceleration and production of almost monoenergetic protons have been observed using a linearly polarized laser focused into a supersonic hydrogen gas jet. A recent experiment [42] has also produced monoenergetic protons of $4 \%$ energy spread using circularly polarized laser and a hydrogen gas target.

\section{Acknowledgments}

Discussions with Bob Bingham (Rutherford Appleton Laboratory) and Francesco Pegoraro (University of Pisa) are gratefully acknowledged. The helpful comments by the anonymous Referee are appreciated.

[1] Bulanov, S. V., Esirkepov, T. Zh., Khoroshkov, V. S., Kuznetsov, A. V. and Pegoraro, F. Oncological hadrontherapy with laser ion accelerators, Phys. Lett. A 299, 240-247 (2002).

[2] K W D Ledingham, W Galster, and R Sauerbrey, Laser-driven proton oncology - a unique new cancer therapy? Br. J. Radiol. 80, 855-858 (2007)

[3] Linz, U. and Alonso, J. What will it take for laser driven proton accelerators to be applied to tumor therapy? Phys. Rev. STAB 10, 094801 (2007).

[4] M. Roth, T. E. Cowan, M. H. Key, S. P. Hatchett, C. Brown, W. Fountain, J. Johnson, D. M. Pennington, R. A. Snavely, S. C. Wilks, K. Yasuike, H. Ruhl, F. Pegoraro, S. V. Bulanov, E. M. Campbell, M. D. Perry, and H. Powell, Fast ignition by intense laser-accelerated proton beams, Phys. Rev. Lett. 86, 436439 (2001)

[5] Mora P 2007 Laser driven ion acceleration AIP Conf. Proc. 920 98-117

[6] A Macchi, A Sgattoni, S Sinigardi, M Borghesi, and M Passoni, Advanced strategies for ion acceleration using high-power lasers, Plasma Phys. Control. Fusion 55, 124020(10pp) (2013).

[7] A. Sgattoni, M. Tamburini, T. V. Liseykina, A. S. Nindrayog, F, Pegoraro, and A. Macci, Simulation studies of radiation pressure-driven light sail and shock acceleration, Proc. SPIE 8779, 87790H, doi:10.1117/12.2017379 (2013).

[8] Krushelnick K, E. L. Clark, Z. Najmudin, M. Salvati, M. I. K. Santala, M. Tatarakis, A. E. Dangor, V. Malka, D. Neely, R. Allott, and C. Danson 1999 Multi-MeV ion production from high-intensity laser interactions with underdense plasmas, Phys. Rev. Lett. 83 737-740

[9] M. S. Wei, S. P. D. Mangles, Z. Najmudin, B.Walton, A. Gopal, M. Tatarakis, A. E. Dangor, E. L. Clark, R. G. Evans, S. Fritzler, R. J. Clarke, C. Hernandez-Gomez, D. Neely, W. Mori, M. Tzoufras, and K. Krushelnick, Ion acceleration by collisionless shocks in high-intensity-laser-underdense-plasma interaction, Phys. Rev. Lett. 93, 155003 (2004).

[10] Y. Sentoku, T. V. Liseikina, T. Zh. Esirkepov, F. Califano, N. M. Naumova, Y. Ueshima, V. A. Vshivkov, Y. Kato, K. Mima, K. Nishihara, F. Pegoraro, and S. V. Bulanov, High density collimated beams of relativistic ions produced by petawatt laser pulses in plasmas, Phys. Rev. E 62, 7271-7281 (2000).

[11] S. P. Hatchett, C. G. Brown, T. E. Cowan, E. A. Henry, J. S. Johnson, M. H. Key, J. A. Koch, A. B. Langdon, B. F. Lasinski, R. W. Lee, A. J. Mackinnon, D. M. Pennington, M. D. Perry, T. W. Phillips, M. Roth, T. C. Sangster, M. S. Singh, R. A. Snavely, M. A. Stoyer, S. C. Wilks, and K. Yasuike, Electron, photon, and ion beams from the relativistic interaction of Petawatt laser pulses with solid targets, Phys. Plasmas 7, 2076-2082 (2000).

[12] S. C. Wilks, A. B. Langdon, T. E. Cowan, M. Roth, M. Singh, S. Hatchett, M. H. Key, D. Pennington, A. MacKinnon, and R. A. Snavely, Energetic proton generation in ultra-intense lasersolid interactions, Phys. Plasmas 8, 542-549 (2001). 
[13] S. V. Bulanov, T. Zh. Esirkepov, F. F. Kamenets, Y. Kato, A. V. Kuznetsov, K. Nishihara, F. Pegoraro, T. Tajima, and V. S. Khoroshkov, Generation of high-quality charged particle beams during the acceleration of ions by high-power laser radiation, Plasma Phys. Rep. 28, 975-991 (2002).

[14] Q. L. Dong, Z. M. Sheng, M. Y. Yu, and J. Zhang, Optimization of ion acceleration in the interaction of intense femtosecond laser pulses with ultrathin foils, Phys. Rev. E 68, 026408 (2003).

[15] C. T. Zhou and X. T. He, Influence of a large oblique incident angle on energetic protons accelerated from solid-density plasmas by ultraintense laser pulses, Appl. Phys. Lett. 90, 031503 (2007).

[16] L. Robson, P. T. Simpson, R. J. Clarke, K. W. D. Ledingham, F. Lindau, O. Lundh, T. McCanny, P. Mora, D. Neely, C.-G. Wahlström, M. Zepf, and P. McKenna, Scaling of proton acceleration driven by petawatt-laser-plasma interactions, Nat. Phys. 3, 58-62 (2007).

[17] K. Lee, S. H. Park, Y.-H. Cha, J. Y. Lee, Y. W. Lee, K.-H. Yea, and Y. U. Jeong, Generation of intense proton beams from plastic targets irradiated by an ultraintense laser pulse, Phys. Rev. E 78, 056403 (2008).

[18] M. Passoni and M. Lontano, Theory of light-ion acceleration driven by a strong charge separation, Phys. Rev. Lett. 101, 115001 (2008).

[19] P. Antici, J. Fuchs, E. d'Humières, J. Robiche, E. Brambrink, S. Atzeni, A. Schiavi, Y. Sentoku, P. Audebert, and P. Pépin, Laser acceleration of high-energy protons in variable density plasmas, New J. Phys. 11, 023038 (2009)

[20] T. Esirkepov, M. Borghesi, S. V. Bulanov, G. Mourou, and T. Tajima, Highly efficient relativistic-ion generation in the laser-piston regime, Phys. Rev. Lett. 92, 175003 (2004).

[21] X. Q. Yan, C. Lin, Z. M. Sheng, Z. Y. Guo, B. C. Liu, Y. R. Lu, J. X. Fang, and J. E. Chen, Generating high-current monoenergetic proton beams by a circularly polarized laser pulse in the phase-stable acceleration regime, Phys. Rev. Lett. 100, 135003 (2008).

[22] O. Klimo, J. Psikal, J. Limpouch, and V. T. Tikhonchuk, Monoenergetic ion beams from ultrathin foils irradiated by ultrahigh-contrast circularly polarized laser pulses, Phys. Rev. ST Accel. Beams 11, 031301 (2008).

[23] V. K. Tripathi, C. S. Liu, X. Shao, B. Eliasson, and R. Z. Sagdeev, Laser acceleration of monoenergetic protons in a self-organized double layer from thin foil, Plasma Phys. Controlled Fusion 51, 024014 (2009).

[24] A. P. L. Robinson, M. Zepf, S. Kar, R. G. Evans, and C. Bellei, Radiation pressure acceleration of thin foils with circularly polarized laser pulses, New J. Phys. 10, 013021 (2008).

[25] B. Eliasson, C. S. Liu, X. Shao, R. Z. Sagdeev, and P. K. Shukla, Laser acceleration of monoenergetic protons via a double layer emerging from an ultra-thin foil, New J. Phys. 11, 073006 (2009).

[26] E. Ott, Nonlinear evolution of the Rayleigh-Taylor instability of a thin layer, Phys. Rev. Lett. 29, 1429-1432 (1972).

[27] F. Pegoraro and S. V. Bulanov, Photon bubbles and ion acceleration in a plasma dominated by the radiation pressure of an electromagnetic pulse, Phys. Rev. Lett. 99, 065002 (2007).

[28] T.-C. Liu, X. Shao, C.-S. Liu, J.-J. Su, B. Eliasson, V. Tripathi, G. Dudnikova, and R. Z. Sagdeev, Energetics and energy scaling of quasi-monoenergetic protons in laser radiation pressure acceleration, Phys. Plasmas 18, 123105 (2011).

[29] T.-C. Liu, X. Shao, C.-S. Liu, M. He, B. Eliasson, V. Tripathi, J.-J. Su, J. Wang, and S.-H. Chen, Generation of quasimonoenergetic protons from thin multi-ion foils by a combination of laser radiation pressure acceleration and shielded Coulomb repulsion, New J. Phys. 15, 025026 (2013).

[30] T.-C. Liu, X. Shao, C.-S. Liu, B. Eliasson, J. Wang, and S.-H. Chen, Enhancement of proton energy by polarization switch in laser acceleration of multi-ion foils, Phys. Plasmas 20, 103112 (2013)

[31] R. Z. Sagdeev, Cooperative phenomena and shock waves in collisionless plasmas, in M. A. Lentovich (ed.) Rev. Plasma Phys., Vol. 4 (Consultants Bureau, New York, 1966), pp. 23-91.

[32] D. W. Forslund and J. P. Freidberg, Theory of Laminar Collisionless Shocks, Phys. Rev. Lett. 27, 1189-1192 (1971).

[33] D. A. Tidman and N. A. Krall, Shock waves in collisionless plasmas (Wiley Interscience, New York, 1971).

[34] F. W. Perkins and Y. C. Sun, Double layers without current, Phys. Rev. Lett. 46(2), 115-118 (1981).

[35] P. Michelsen and J. J. Rasmussen (eds.) Symposium on Plasma Double Layers, 16-18 June 1982, Report RISØ-R-472, Ris $\varnothing$ National Laboratory (Roskilde, Denmark, September 1982). http://www.risoe.dk/rispubl/reports_INIS/RISOR472.pdf

[36] H. Schamel, Kinetic theory of phase space vortices and double layers, Phys. Scr. T2A, 228-237 (1982).

[37] H. Schamel, Weak double layers: Existence, stability, evidence, Z. Naturforsch. A 38, 1170-1183 (1983).

[38] H. Schamel, Electron holes, ion holes and double layers, Phys. Rep. 140(3), 161-191 (1986).

[39] H. Schamel and S. Bujarbarua, Analytical double layers, Phys. Fluids 26, 190-193 (1983).

[40] K. S. Goswami, K. Saharia and H. Schamel, Theory of current-free double layers in plasmas, Phys. Plasmas 15, 062111 (2008).

[41] L. O. Silva, M. Marti, J. R. Davies, R. A. Fonseca, C. Ren, F. S. Tsung, and W. B. Mori, Proton shock acceleration in laser-plasma interactions, Phys. Rev. Lett. 92, 015002 (2004).

[42] C. A. J. Palmer, N. P. Dover, I. Pogorelsky, M. Babzien, G. I. Dudnikova, M. Ispiriyan, M. N. Polyanskiy, J. Schreiber, P. Shkolnikov, V. Yakimenko, and Z. Najmudin, Monoenergetic proton beams accelerated by a radiation pressure driven shock, Phys. Rev. Lett. 106, 014801 (2011).

[43] D. Haberberger, S. Tochitsky, F. Fiuza, C. Gong, R. A. Fonseca, L. O. Silva, W. B. Mori, and C. Joshi, Collisionless shocks in laser-produced plasma generate monoenergetic high-energy proton beams, Nature Phys. 8, 95-99 (2012)

[44] M.-Q. He, X. Shao, C.-S. Liu, T.-C. Liu, J.-J. Su, G. Dudnikova, R. Z. Sagdeev, and Z.-M. Sheng, Quasi-monoenergetic protons accelerated by laser radiation pressure and shocks in thin gaseous targets, Phys. Plasmas 19, 073116 (2012).

[45] R. A. Cairns, R. Bingham, P. Norreys, and R. Trines, Laminar shocks in high power laser plasma interactions, Phys. Plasmas 21, 022112 (2014).

[46] A. Macchi, A. S. Nindrayog, and F. Pegoraro, Solitary versus shock wave acceleration in laser-plasma interactions, Phys. 
Rev. E 85, 046402 (2012).

[47] G. Sorasio, M. Marti, R. Fonseca and L. O. Silva, Very High Mach-Number Electrostatic Shocks in Collisionless Plasmas, Phys. Rev. Lett. 96, 045005 (2006).

[48] F. Fiuza, A. Stockem, E. Boella, R. A. Fonseca, L. O. Silva, D. Haberberger, S. Tochitsky, C. Gong, W. B. Mori and C. Joshi, Phys. Rev. Lett. 109, 215001 (2012).

[49] A. Stockem, E. Boella, F. Fiuza and L. O. Silva, Relativistic generalization of formation and ion-reflection conditions in electrostatic shocks, Phys. Rev. E 87, 043116 (2013).

[50] F. Fiuza, A. Stockem, E. Boella, R. A. Fonseca, L. O. Silva, D. Haberberger, S. Tochitsky, W. B. Mori and C. Joshi, Ion acceleration from laser-driven electrostatic shocks, Phys. Plasmas 20, 056304 (2013).

[51] H. Schamel, Stationary solitary, snoidal and sinusoidal ion acoustic waves, Plasma Phys. 14, 905 (1972).

[52] S. B. Bujarbarua and H. Schamel, Theory of finite-amplitude electron and ion holes, J. Plasma Phys. 25(03), 515-529 (1981).

[53] K. Y. Kim, Weak monotonic double layers, Phys. Lett. A 97, 45-50 (1983).

[54] B. D. Fried and S. D. Conte, The Plasma Dispersion Function (Academic Press, New York, 1961).

[55] H. Schamel and S. Bujarbarua, Solitary plasma hole via ion-vortex distribution, Phys. Fluids 23, 2498-2499 (1980). 